\title{
Efecto de la competencia digital docente en el uso del blended learning en formación profesional
}

\author{
Jesús López-Belmonte* \\ Antonio José Moreno-Guerrero** \\ Santiago Pozo-Sánchez ${ }^{\star \star \star}$ \\ Juan Antonio López-Núñez**
}

Artículo recibido:

28 de julio de 2019

Artículo aceptado:

4 de octubre de 2019

Artículo de investigación

\section{Resumen}

La proliferación de la tecnología educativa en la formación profesional ha llevado a que el colectivo docente que desarrolla su cometido en dicha etapa tenga que disponer de un adecuado nivel de destrezas digitales para hacer un uso eficaz de metodologías innovadoras. El estudio que se presenta tiene como objetivo conocer la influencia de la competencia digital en el uso del blended learning en el profesorado de formación profesional. Para ello se ha seguido un método de investigación cuantitativo de tipo descriptivo y correlacional,

* Departamento de Educación, Universidad Internacional de Valencia, España jesus.lopezb@campusviu.es

** Departamento de Didáctica y Organización Escolar, Universidad de Granada, España ajmoreno@ugr.es_juanlope@ugr.es INVESTIGACIÓN BIBLIOTECOLÓGICA, vol. 34, núm. 83, abril/junio, 2020, México, ISSN:
2448-8321 pp. 187-205 
en una muestra de 627 docentes españoles. Como instrumento de recogida de datos se utilizó un cuestionario. Los resultados revelan un déficit competencial en las distintas áreas de la competencia digital, hecho que repercute en el uso del blended learning en la etapa de formación profesional y que los propios docentes perciben como motivo principal, concluyendo que tal competencia influye en la utilización de este enfoque emergente.

Palabras clave: Tecnología de la Información; Innovación Educacional; Aprendizaje Combinado; Competencia Digital

Effect of digital teaching competence in the use of blended learning in Vocational Training

Jesús López-Belmonte, Antonio José Moreno-Guerrero, Santiago Pozo-Sánchez and Juan Antonio López-Núñez

\section{Abstract}

The proliferation of educational technology in Vocational Training has meant that the teaching group that develops its mission at that stage must have an adequate level of digital skills to make an effective use of innovative methodologies. The study presented aims to know the influence of digital competence in the use of blended learning in Vocational Training teachers. For that reason, a quantitative research method of descriptive and correlational type has been followed, with a sample of 627 Spanish teachers. As a data collection instrument, a questionnaire was used whose results reveal a competence deficit in the different areas of digital ability, a fact that affects the use of blended learning in the vocational training stage, and that teachers themselves perceive as the main reason to conclude that such competence influences the use of this emerging approach.

Keywords: Information Technology; Educational Innovations; Blended Learning; Digital Competence 


\section{INTRODUCCIÓN}

A ctualmente, las tecnologías de la información y comunicación (TIC) educativo, donde se están extendiendo cada vez más (Larionova et al., 2018). Esta situación desemboca en una transformación en el sistema educativo con la finalidad de atender a las necesidades de los discentes de una sociedad en constante cambio (Viñals Blanco y Cuenca Amigo, 2016) y acostumbrados a vivir rodeados de tecnología (Rodríguez Licea, López Frías y Mortera Gutiérrez, 2017). Esto provoca nuevas exigencias entre los docentes (Area Moreira, 2014), que consideran una necesidad el poseer un nivel adecuado de competencia digital para aplicar procesos de enseñanza-aprendizaje innovadores (López Belmonte et al., 2019; Elche Larranaga y Yubero, 2019) y fomentar la inclusión de la tecnología educativa en los espacios de aprendizaje (Area Moreira, Hernández Rivero y Sosa Alonso, 2016).

Dadas estas circunstancias, se hace necesaria la adquisición de una serie de competencias por parte de los docentes, hecho que la propia Unión Europea recomendó en el Parlamento Europeo y en el Consejo sobre competencias clave para el aprendizaje permanente en 2006 (Rodríguez García, Romero Rodríguez y Agreda Montoro, 2019).

Dentro de las competencias clave que proponen se halla la competencia digital, entendida como una de las destrezas necesarias para el despliegue de una educación innovadora propia del nuevo milenio (Rodríguez García, Trujillo Torres y Sánchez Rodríguez, 2019), donde las TIC han asumido un gran protagonismo (Castañeda, Esteve y Adell, 2018). Asimismo, se trata de una competencia dinámica, pues la tecnología es concebida como un constructo variable que se encuentra en constante estado de renovación y actualización (Avitia Carlos y Uriarte Ramírez, 2017).

La competencia digital docente se estructura en diversas áreas (1. Información y alfabetización informacional; 2. Comunicación y colaboración; 3. Creación de contenidos digitales; 4. Seguridad; 5. Resolución de problemas), establecidas dentro del marco común de competencia digital docente, desarrollada en 2017 por el Instituto Nacional de Tecnología Educativa y de Formación del Profesorado (INTEF, 2017).

Las propias administraciones educativas españolas siguen las recomendaciones de las propuestas europeas estableciendo programas para el desarrollo de la competencia digital en el ámbito educativo, tanto para los docentes como para los discentes (Prendes Espinosa y Gutiérrez Porlán, 2013; Rodríguez-García, Raso Sanchez y Ruiz-Palmero, 2019), teniendo presentes factores influyentes para su desarrollo como la edad, el sexo, la experiencia 
profesional, el uso de recursos tecnológicos, el tipo de escuela y el desempeño de cargos académicos (Arrosagaray et al., 2019; García Martín y Cantón Mayo, 2019).

Sobre el nivel competencial en el plano tecnopedagógico, los propios docentes muestran ciertos desequilibrios en las habilidades concernientes a cada área de la competencia digital (Fernández Cruz, Fernández Díaz y Rodríguez Mantilla, 2018; Fuentes Cabrera, López Belmonte y Pozo Sánchez, 2019), dificultando la tarea de integración de la tecnología desde una óptica pedagógica (Cela Ranilla et al., 2017). Todo ello viene motivado por una falta de cualificación y reciclaje de conocimientos que son necesarios para efectuar una acción formativa contemporánea (Prendes Espinosa, Gutiérrez Porlán y Martínez Sánchez, 2018).

Hoy día es común que las actividades educativas tradicionales incluyan la utilización de tecnologías móviles, propiciando el desarrollo de la alfabetización digital (Aarsand, 2019) y la combinación entre métodos clásicos y el uso de las TIC, generando distintos espacios de aprendizaje (Nogueira Leite, Shigueo Hoji y Abdala Júnior, 2018) que llevan a los estudiantes a vivenciar verdaderas experiencias de aprendizaje (Cabero Almenara y Barroso Osuna, 2018). Esto es lo que se conoce como blended learning, un enfoque metodológico que fomenta el uso de las TIC, herramientas con las que se puede integrar una plataforma electrónica y crear un ambiente de aprendizaje combinando las clases presenciales con entornos virtuales (Carmona Herrera, Cruz Rosales y García Martínez, 2019).

En los últimos tiempos, la enseñanza apoyada en medios digitales ha cobrado especial importancia para el desarrollo de los procesos formativos (Moreno Guerrero, 2019), sobre todo en la Formación Profesional (Dummert, Leber y Schwengler, 2019). Esta es una de las etapas educativas que más cambios e investigaciones ha ido experimentando (Wheelahan, 2015), siendo entendida como un elemento esencial para el desarrollo, la transformación, la equidad y la inclusión social (Sarmiento Espinel, Silva Arias y van Gameren, 2019).

Por su parte, las investigaciones sobre el uso de blended learning en la Formación Profesional muestran que la aplicación de este método de enseñanza conlleva un alto nivel de aceptación por parte de los alumnos (Gómez Zermeno, Alemán de la Garza y Figueroa Orejarena, 2019; Pfefferle, Van den Stock y Nauerth, 2010), debido a los buenos resultados obtenidos en su formación (De Lange et al., 2018) como consecuencia del papel activo por parte del estudiante, promoviéndose el trabajo en equipo, el liderazgo, la autoconfianza, la autoestima (Nogueira Leite, Shigueo Hoji y Abdala Júnior, 2018) y la competencia digital (Tsai y Lee, 2012). 
En estos nuevos entornos formativos, el profesorado asume un papel de guía (Gómez Zermeno, Alemán de la Garza y Figueroa Orejarena, 2019), tratando de combinar los elementos prácticos y teóricos (Nogueira Leite, Shigueo Hoji y Abdala Júnior, 2018) y adecuar los diferentes estilos de aprendizaje a las singularidades de los estudiantes (González Bello, Valenzuela Miranda y González Beltrones, 2015). Por ello, su formación es crucial (Ruhalahti, Korhonen y Rasi, 2017), ya que carencias competenciales en el uso del blended learning puede generar una praxis docente insuficiente que limite las potencialidades de dicho enfoque (Bliuc et al., 2012).

\section{JUSTIFICACIÓN Y OBJETIVOS DEL ESTUDIO}

Dada la transcendencia que han alcanzado las TIC y las metodologías emergentes en los procesos de enseñanza-aprendizaje, surge este estudio con el propósito de indagar en el enfoque formativo blended learning desde una perspectiva competencial del profesorado en el plano digital.

Se realizó una aproximación sobre el estado de la cuestión en bases de datos -como Scopus y Web of Science- revelando que, a pesar de ser dos temáticas con un nivel alto de tratamiento, existe un déficit en investigaciones que busquen una asociación entre el uso del blended learning y la competencia digital docente en la Formación Profesional, analizándose siempre por separado.

Por tanto, este estudio se desarrolla para solventar esta brecha con el objetivo general de conocer si el nivel de competencia digital docente influye en la utilización del enfoque didáctico blended learning. De esta forma, como objetivos específicos derivados del anterior se pretende concretar el grado de utilización del blended learning en el profesorado analizado, descubrir los motivos de la no utilización del blended learning y averiguar la incidencia sobre dicho enfoque del nivel en cada una de las cinco áreas que articulan la competencia digital.

\section{MÉTodo}

\section{Diseño de investigación}

El estudio que se presenta se aborda desde una visión exploratoria con la finalidad de iniciar la senda investigativa de ambos conceptos interconectados. Para ello y para lograr la consecución de los objetivos formulados se ha empleado un diseño de investigación de naturaleza descriptiva y correlacional a través de una metodología cuantitativa. 


\section{Muestra}

La selección se llevó a cabo por medio de un muestreo aleatorio simple, tomando como referencia la base de datos del Ministerio de Educación y Formación Profesional (https://bit.ly/2TzPFiY). Se seleccionaron primeramente 2650 docentes, pertenecientes a 530 centros con ciclos formativos de Formación Profesional, de los que se obtuvo una participación de $23.66 \%$ ( $n=627)$. El $73.52 \%$ eran hombres y $26.48 \%$ mujeres, ambos con edades comprendidas entre 28 y 61 años $(\mathrm{Me}=43 ; \mathrm{DT}=11.82)$.

\section{Instrumento}

El proceso de recolección de datos se realizó mediante un cuestionario ad hoc diseñado con base en otros instrumentos validados sobre el arte que abarca el estudio, como los de Agreda Montoro, Hinojo Lucena y Sola Reche (2016) y Tourón et al. (2018).

Dicho cuestionario se compone de 66 cuestiones, clasificadas en seis dimensiones (Tabla 1), con un formato de respuesta variado: de escala Likert, de elección cerrada, de opinión abierta y dicotómicas.

La validación del instrumento se realizó desde varias aproximaciones. Se empleó el método Delphi para obtener un feedback objetivo y anónimo de un grupo de expertos compuesto por seis doctores en tecnología educativa de distintas universidades españolas, cuyas objeciones fueron tenidas en cuenta para la optimización de la herramienta y estudiadas a nivel estadístico para verificar si las retroalimentaciones se asocian, son concordantes y pertinentes. Ello se materializó mediante las pruebas Kappa de Fleiss $(\mathrm{K}=0.81)$ y W de Kendall $(\mathrm{W}=0.84)$, obteniéndose valores adecuados. También se realizó un análisis factorial exploratorio por medio del método de componentes principales, aplicando una rotación varimax. Asimismo, entre las variables en la prueba de esfericidad de Bartlett $(\mathrm{PEB}=2765.37 ; p<0.001)$ se reportó dependencia, y adecuación muestral con el test de Kaiser-Meyer-Olkin (KMO = 0.92).

La fiabilidad del cuestionario se decretó por medio de los estadísticos alfa $(\alpha)$ de Cronbach, la fiabilidad compuesta (FC) y la varianza media extractada (VME), obteniéndose adecuados índices de consistencia interna (Tabla 1). 


\begin{tabular}{|l|c|c|c|}
\hline \multicolumn{1}{|c|}{ Dimensión } & $\boldsymbol{\alpha}$ & FC & VME \\
\hline Socioeducativa & 0.86 & 0.89 & 0.74 \\
\hline Información y alfabetización informacional & 0.84 & 0.88 & 0.79 \\
\hline Comunicación y colaboración & 0.87 & 0.91 & 0.83 \\
\hline Creación de contenidos digitales & 0.85 & 0.82 & 0.77 \\
\hline Seguridad & 0.87 & 0.84 & 0.81 \\
\hline Resolución de problemas & 0.82 & 0.75 & 0.66 \\
\hline
\end{tabular}

Tabla 1. Organización dimensional y resultados de fiabilidad

Fuente: elaboración propia

\section{Variables de estudio}

A continuación, se muestran las codificaciones de cada una de las variables empleadas:

FRBL: Frecuencia de utilización del blended learning;

ÁREA1: Nivel de destrezas en información y alfabetización digital;

ÁREA2: Nivel de destrezas en comunicación y colaboración;

ÁREA3: Nivel de destrezas en creación de contenidos digitales;

ÁREA4: Nivel de destrezas en seguridad;

ÁREA5: Nivel de destrezas en resolución de problemas;

DFCD: Déficit formativo en competencia digital;

FCBL: Falta de confianza en el blended learning;

ART: Ausencia de recursos tecnológicos;

ENCA: Enfoque no compatible con el alumnado;

NTIC: Negativa hacia el uso de las TIC en el proceso de instrucción.

\section{Procedimiento}

La investigación comenzó en 2018. En primer lugar, se produjo el diseño y validación del cuestionario. Una vez optimizado el instrumento, se procedió a la selección de la muestra tras la consulta en la base de datos del Ministerio de Educación y Formación Profesional. El cuestionario se envió por correo electrónico a los distintos centros educativos resultantes tras el proceso de muestreo. La fase de cumplimentación del mismo se prolongó durante cinco meses, tiempo durante el cual los investigadores se encontraron disponibles para resolver cualquier incidencia o duda en la recogida de datos. Transcurrido el periodo, se produjo la extracción de datos y su preparación con la 
intención de realizar un análisis profundo y pertinente que permitiera dar respuesta a los objetivos planteados en el estudio.

\section{Análisis de datos}

El tratamiento estadístico se realizó a través del programa Statistical Package for the Social Sciences v.24 (SPSS), tomando como diferencias estadísticamente significativas aquellos valores de $p<0.05$.

En el despliegue estadístico descriptivo se ha empleado la media (M), la desviación típica (DT), el coeficiente de asimetría de Pearson (CAP) y el de apuntamiento de Fisher (CAF) para determinar la tendencia de la distribución muestral.

Para la clasificación de los sujetos se ha empleado un análisis clúster de tipo bietápico (margen de error $=5 \%$ ), para realizar una doble agrupación de los participantes en grupos similares procedentes de un conjunto aparentemente heterogéneo, estableciéndose cinco conglomerados para el nivel de la competencia digital docente y cuatro conglomerados para la frecuencia de utilización del blended learning.

La comparación de variables se ha efectuado mediante el test Chi-cuadrado de Pearson $\left(\chi^{2}\right)$, complementado con la V de Cramer (V) y el coeficiente de contingencia (Cont) para determinar el grado de fortaleza de la asociación.

\section{Resultados}

El presente apartado recoge los principales hallazgos encontrados en el proceso investigador. La Figura 1 refleja los resultados sobre el uso del blended learning en los docentes analizados. Destacan los dos extremos de la distribución, el izquierdo por reflejar que más de la mitad de la muestra no utiliza este enfoque innovador en los procesos de enseñanza-aprendizaje, y el derecho por aglutinar una mínima cifra de docentes que sí emplean tal metodología emergente. Asimismo, los valores centrales son compartidos -con puntuaciones similares- por aquellos docentes que usan el blended learning, tanto de manera esporádica como con frecuencia, siendo ambas cifras no superiores a la cuarta parte porcentual de los datos reportados. 


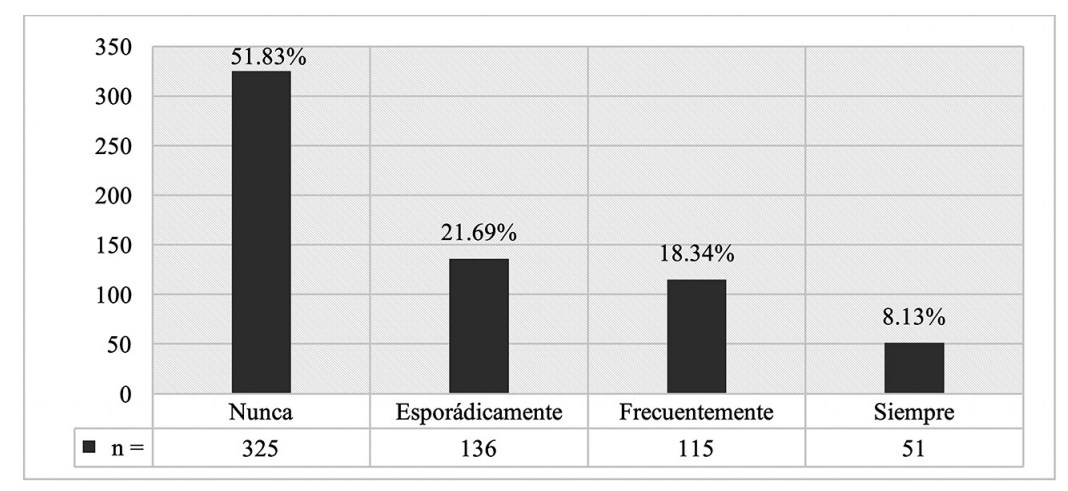

Figura 1. Utilización del enfoque blended learning (FRBL) en el profesorado Fuente: elaboración propia

En cuanto a las razones manifestadas por el profesorado para justificar el nulo empleo del blended learning como enfoque didáctico (Figura 2), un subgrupo de 325 docentes revelaron negativa ante su uso, aludiendo -principalmente- a la falta de formación en el terreno tecnopedagógico (45.23 \%). Otros de los motivos postulados es la falta de confianza hacia el enfoque innovador $(22.15 \%)$, una postura contraria hacia el uso de las TIC (17.23 \%), la ausencia de recursos tecnológicos para desplegar tal metodología (12 \%) y la incompatibilidad con el alumnado existente (3.38\%).

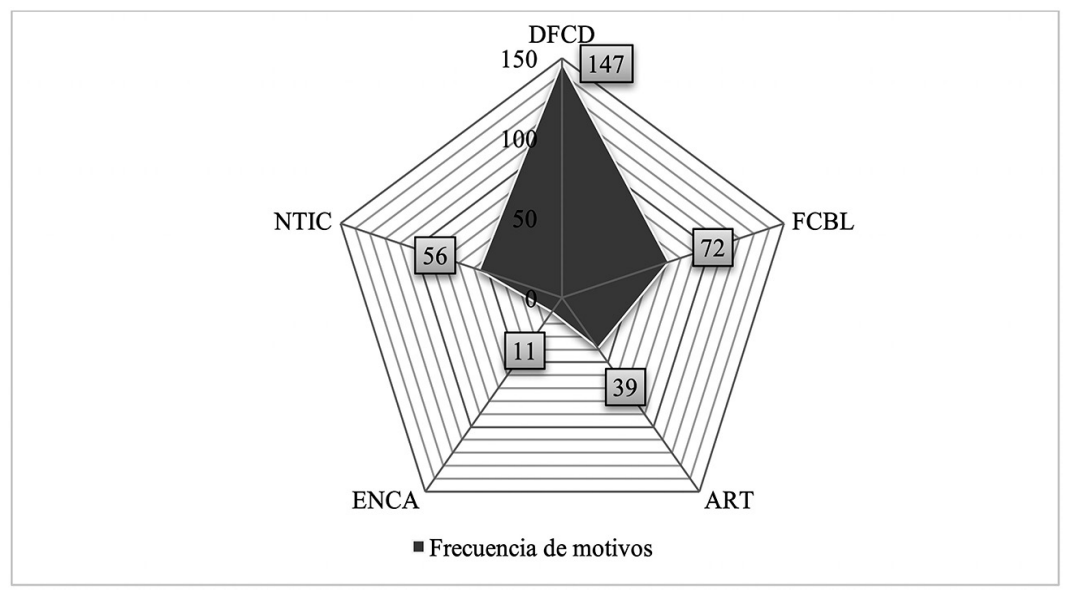

Figura 2. Motivos por los que no se utiliza el enfoque blended learning Fuente: elaboración propia 
En la Tabla 2 se recoge el nivel de destrezas del profesorado en el ámbito digital. Los resultados obtenidos revelan carencias competenciales en las distintas áreas de la competencia digital. Del mismo modo, se aprecia una distribución asimétrica tendente a la izquierda, dada la concentración de los datos en los rangos más deficientes de la escala. Las puntuaciones más elevadas se encuentran en las áreas de la información y alfabetización informacional y de la comunicación y colaboración del profesorado. A pesar de ello, sus valores se concentran en el $\mathrm{R}=2$ de la escala (nivel bajo). El resto de áreas reflejan puntuaciones deficientes cercanas al segundo rango de medición competencial. La representación visual de las puntuaciones medias (Figura 3) muestra una media totalizada que sitúa el grado de competencia digital de estos profesionales en un nivel bajo, lo que desemboca en un grave déficit en materia tecnopedagógica que no logra alcanzar los valores óptimos deseados -establecidos a partir del rango intermedio de la escala de medición- para efectuar una praxis innovadora de manera adecuada.

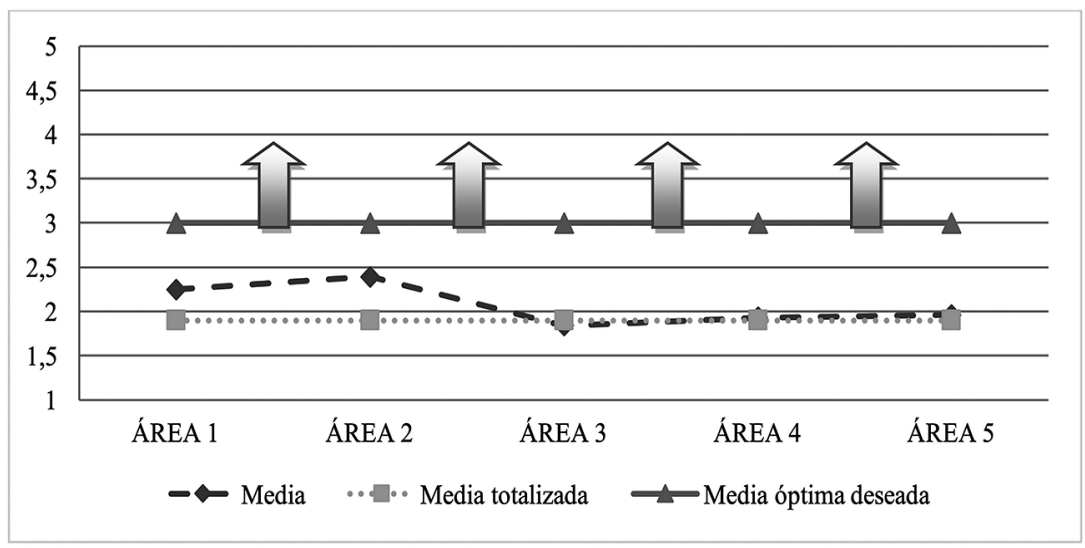

Figura 3. Comparación de puntuaciones medias

Fuente: elaboración propia

Por último, se presentan los resultados alcanzados en el análisis correlacional entre la frecuencia de utilización del blended learning y las distintas áreas de la competencia digital (Tabla 3). Se han encontrado diferencias estadísticamente significativas en las áreas relativas a la comunicación y colaboración y a la seguridad en el plano digital $(p<0.05)$, con fuerza de asociación moderada, siendo superior en el área de la seguridad. Estos hallazgos manifiestan que los docentes que disponen de mayores destrezas en tales áreas son más propensos a desarrollar un proceso formativo a través del enfoque blended learning en su praxis diaria. Por otra parte, el resto de áreas no han alcanzado significancia estadística, no hallándose vinculación entre la frecuencia de uso y el nivel de destrezas. 


\begin{tabular}{|c|c|c|c|c|c|c|c|}
\hline \multirow{4}{*}{ 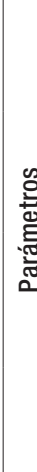 } & 岕 & \begin{tabular}{l}
\multirow{+}{0}{} \\
0 \\
0 \\
0 \\
1
\end{tabular} & 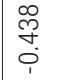 & $\begin{array}{l}0 \\
\stackrel{\Omega}{0} \\
0 \\
0\end{array}$ & $\begin{array}{l}0 \\
\circ \\
0 \\
1\end{array}$ & $\begin{array}{l}\overline{8} \\
\overline{1}\end{array}$ & $\begin{array}{l}\overline{5} \\
\infty \\
0 \\
1\end{array}$ \\
\hline & 这 & $\stackrel{\bar{m}}{\rightleftarrows}$ & ্ֻలি & $\begin{array}{l}\hat{\theta} \\
\ddot{0} \\
0\end{array}$ & $\begin{array}{l}\stackrel{\mathscr{R}}{\stackrel{R}{\rho}} \\
\stackrel{0}{0}\end{array}$ & $\begin{array}{l}\stackrel{0}{0} \\
\stackrel{0}{0}\end{array}$ & $\begin{array}{l}\mathscr{0} \\
0 \\
0 \\
0\end{array}$ \\
\hline & 占 & 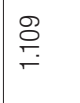 & 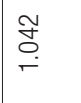 & 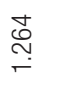 & 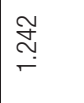 & 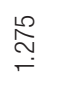 & $\stackrel{\infty}{\stackrel{\infty}{\leftarrow}}$ \\
\hline & $\Sigma$ & $\stackrel{\stackrel{L}{N}}{N}$ & 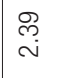 & 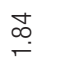 & $\stackrel{\mathscr{O}}{\stackrel{\circ}{-}}$ & $\stackrel{\circ}{\circ}$ & $\stackrel{\leftrightarrow}{\longrightarrow}$ \\
\hline \multirow{6}{*}{ 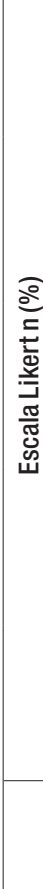 } & 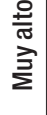 & 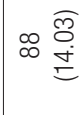 & œ & 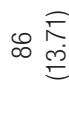 & 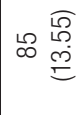 & б & 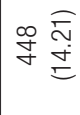 \\
\hline & 온 & 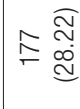 & 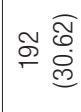 & 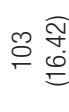 & $\stackrel{\cong}{\cong}$ & 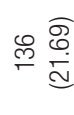 & $\bar{c} \underset{\sim}{\stackrel{\widehat{D}}{\mathscr{d}}}$ \\
\hline & $\begin{array}{l}\frac{\text { 읗 }}{\sum^{\circ}} \\
\end{array}$ & $\underset{\sim}{\stackrel{\widehat{m}}{\sim}}$ & 足 & 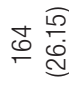 & 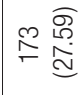 & 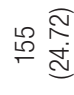 & 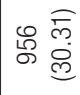 \\
\hline & 옳 & 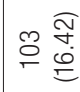 & 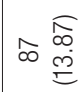 & 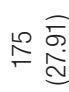 & 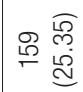 & 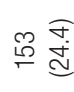 & 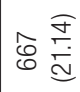 \\
\hline & \multirow[t]{2}{*}{ 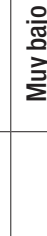 } & 次 & $\widehat{\sim} \stackrel{\widehat{m}}{ \pm}$ & 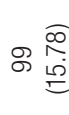 & 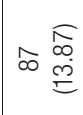 & $\approx$ ๙ & 识 \\
\hline & & 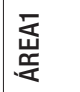 & 妾 & 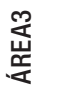 & 离 & 点 & 픙 \\
\hline
\end{tabular}




\begin{tabular}{|c|c|c|c|c|c|c|c|c|c|c|c|c|c|c|c|c|c|c|}
\hline \multirow{4}{*}{ 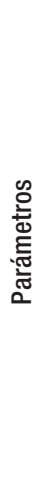 } & $>$ & $\begin{array}{c}\text { ָे } \\
\stackrel{0}{0}\end{array}$ & & & & & & 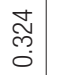 & & & & & & 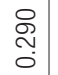 & & & & \\
\hline & 蒿 & $\frac{\bar{\sigma}}{\sigma}$ & & & & & & $\begin{array}{l}\stackrel{+}{\infty} \\
\stackrel{0}{0}\end{array}$ & & & & & & 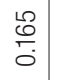 & & & & \\
\hline & $\begin{array}{l}\frac{\overline{0}}{0} \\
\frac{\pi}{3} \\
\vdots \\
\vdots\end{array}$ & 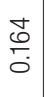 & & & & & & $\begin{array}{l}\infty \\
\tilde{\sigma} \\
0 \\
0\end{array}$ & & & & & & $\stackrel{\stackrel{\infty}{\leftarrow}}{\leftarrow}$ & & & & \\
\hline & 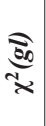 & 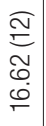 & & & & & & 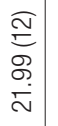 & & & & & & 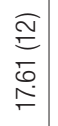 & & & & \\
\hline \multirow{4}{*}{ 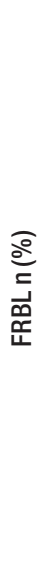 } & 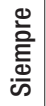 & & $\begin{array}{l}\text { fo } \\
\stackrel{0}{\rho} \\
\text { m }\end{array}$ & 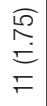 & 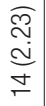 & 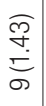 & $\begin{array}{l}\underset{\widetilde{N}}{\mathfrak{d}} \\
\underset{\Xi}{ \pm}\end{array}$ & & 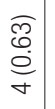 & $\underset{\sim}{\stackrel{E}{E}}$ & 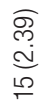 & 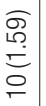 & 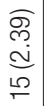 & & 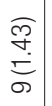 & 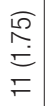 & $\underset{\substack{\widehat{N} \\
\underset{\infty}{\mathcal{E}}}}{ }$ & $\begin{array}{l}\underset{\sigma}{\tilde{E}} \\
\stackrel{ }{\simeq}\end{array}$ \\
\hline & 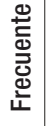 & & $\underset{\Gamma}{E}$ & 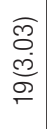 & 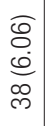 & 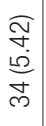 & $\begin{array}{l}\underset{\mathbb{I}}{\mathfrak{d}} \\
\underline{\underline{N}}\end{array}$ & & $\begin{array}{l}\sigma \\
\stackrel{D}{0} \\
0 \\
0\end{array}$ & 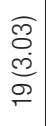 & $\begin{array}{l}\widehat{\sigma} \\
\text { ம. } \\
\hat{n}\end{array}$ & 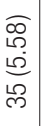 & $\begin{array}{l}\widehat{o} \\
\text { ल. } \\
\text { o }\end{array}$ & & 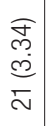 & 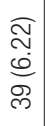 & 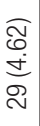 & $\begin{array}{l}\stackrel{\text { }}{+} \\
\stackrel{5}{\sigma}\end{array}$ \\
\hline & 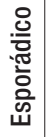 & & 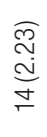 & 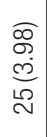 & 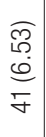 & $\begin{array}{l}\widehat{\sigma} \\
\stackrel{\omega}{\omega} \\
\hat{m}\end{array}$ & $\begin{array}{l}\widehat{\tilde{g}} \\
\stackrel{\rho}{0} \\
\stackrel{\sigma}{\sigma}\end{array}$ & & $\underset{\sim}{\stackrel{\Xi}{E}}$ & 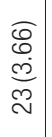 & 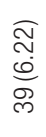 & 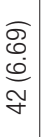 & 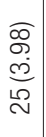 & & 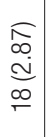 & 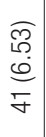 & $\begin{array}{l}\widehat{\sigma} \\
\stackrel{0}{\omega} \\
\hat{\omega}\end{array}$ & 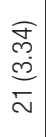 \\
\hline & $\begin{array}{l}\text { 巳્ } \\
\text { 至 }\end{array}$ & & $\begin{array}{l}\text { बे } \\
\text { ले } \\
\bar{\sim}\end{array}$ & $\begin{array}{l}\mathbb{1} \\
\stackrel{0}{\circ} \\
\stackrel{0}{\infty} \\
\stackrel{+}{+}\end{array}$ & 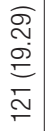 & 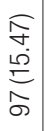 & $\begin{array}{l}\widehat{\theta} \\
\dot{0} \\
\dot{0} \\
\infty \\
0\end{array}$ & & 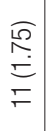 & $\begin{array}{l}\widehat{\mathscr{Q}} \\
\dot{0} \\
\infty \\
\bar{m}\end{array}$ & 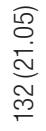 & 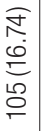 & 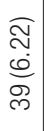 & & $\begin{array}{l}\frac{\sigma}{\infty} \\
\frac{\infty}{\sigma} \\
\frac{\infty}{\infty}\end{array}$ & 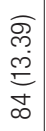 & 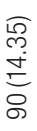 & $\begin{array}{l}\overparen{N} \\
\tilde{\sigma} \\
\bar{\sigma}\end{array}$ \\
\hline & 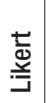 & 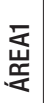 & 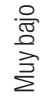 & 욞 & $\begin{array}{l}\frac{0}{0} \\
\frac{0}{2}\end{array}$ & $\frac{9}{\frac{1}{\alpha}}$ & 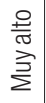 & 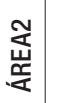 & 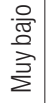 & $\stackrel{\circ}{\stackrel{\circ}{\widetilde{D}}}$ & $\begin{array}{l}\text { 응 } \\
\frac{0}{2}\end{array}$ & 量 & $\begin{array}{l}\frac{0}{\frac{0}{\pi}} \\
\stackrel{3}{\Sigma} \\
\sum\end{array}$ & 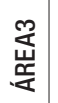 & $\begin{array}{l}\frac{0}{\sqrt{0}} \\
\frac{\pi}{3} \\
\frac{3}{2}\end{array}$ & $\stackrel{\circ}{\mathscr{\widetilde { J }}}$ & $\begin{array}{l}\text { 응 } \\
\frac{\mathbb{d}}{2}\end{array}$ & 운 \\
\hline
\end{tabular}




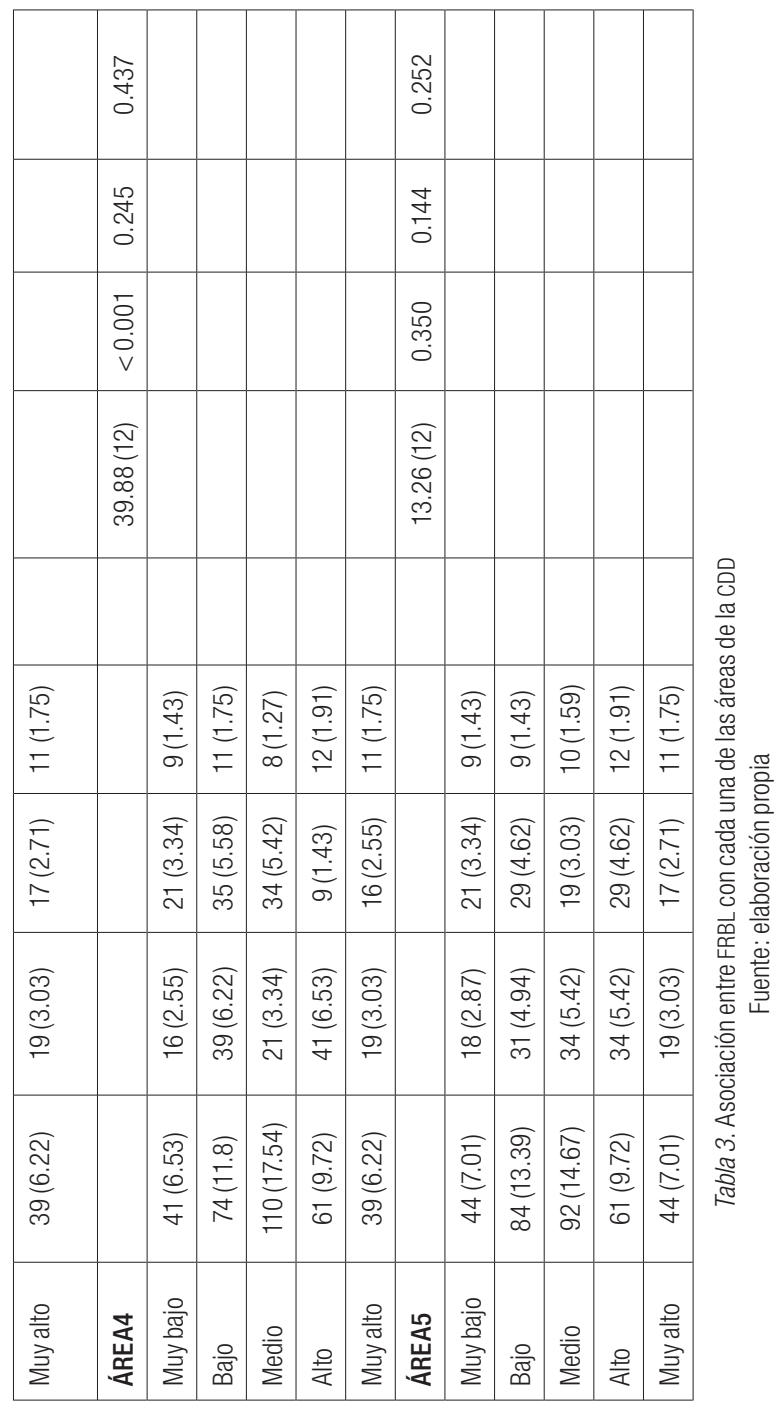




\section{DisCUSióN Y CONCLUSIONES}

La educación para una ciudadanía digital conlleva un ejercicio permanente de renovación del perfil formativo y competencial de los profesionales de la educación. El despliegue del ejercicio docente en una era tecnificada ha obligado a que el profesorado disponga de una competencia digital que le permita desenvolverse en ambientes enriquecidos por la diversidad de recursos, aplicaciones y medios informáticos con los cuales efectuar una praxis innovadora (Elche Larranaga y Yubero, 2019; López Belmonte et al., 2019; Rodríguez García, Trujillo Torres y Sánchez Rodríguez, 2019).

Si bien existen programas que fomentan la mejora de la competencia digital del profesorado (Prendes Espinosa y Gutiérrez Porlán, 2013; Rodríguez-García, Raso Sanchez y Ruiz-Palmero, 2019), los estudios reportados han revelado -en consonancia con los resultados obtenidos en esta investigación- carencias en esta competencia, demostrando que el profesorado aún no dispone de un nivel óptimo para efectuar su cometido laboral de la mano de la tecnología y de manera eficiente (Acebedo Afanador, 2017; Fernández Cruz, Fernández Díaz y Rodríguez Mantilla, 2018; Fuentes Cabrera, López Belmonte y Pozo Sánchez, 2019).

Concretamente, el profesorado de Formación Profesional analizado ha referido poseer mejores destrezas -a pesar de catalogarse como bajas- solamente en las áreas conexas a la información y alfabetización informacional y a la comunicación y colaboración, quedando el resto etiquetadas en un nivel muy deficiente.

Con respecto al uso de metodologías emergentes como el blended learning en la Formación Profesional, los resultados han manifestado que más de la mitad de los sujetos analizados no emplean tal enfoque formativo, justificado -mayoritariamente- en la ausencia de formación para el uso didáctico de la tecnología -como ya asentaron Prendes Espinosa, Gutiérrez Porlán y Martínez Sánchez (2018) - y la falta de confianza en su aplicación. Esta última razón contrasta con los resultados de investigaciones anteriores donde se verifican una serie de ventajas en dicho enfoque como una opinión positiva de los estudiantes (Gómez Zermeno, Alemán de la Garza y Figueroa Orejarena, 2019; Pfefferle, Van den Stock y Nauerth, 2010), resultados académicos satisfactorios (De Lange et al., 2018), así como el fomento de otros indicadores como la participación, la colaboración y la competencia digital de éstos (Nogueira Leite, Shigueo Hoji y Abdala Júnior, 2018; Tsai y Lee, 2012).

Estas potencialidades reflejadas en la literatura sólo atraen a un pequeño grupo de docentes de Formación Profesional a emplear el blended learning en su praxis diaria, demostrándose que aquellos docentes con mejores destrezas tecnopedagógicas son los que más lo emplean, en analogía con Bliuc 
et al. (2012) y Ruhalahti, Korhonen y Rasi (2017), quienes postularon que la baja competencia digital limita los beneficios reportados por un blended learning efectuado por profesionales con amplias destrezas digitales.

En cuanto a las áreas más influyentes en el uso del blended learning, las pruebas estadísticas realizadas determinan que las alusivas a la comunicación y colaboración y a la seguridad son las áreas que más incidencia originan en la utilización de tal metodología innovadora.

Con la realización de este estudio se concluye que el profesorado de la etapa de Formación Profesional no dispone de un nivel de competencia digital óptimo para efectuar un proceso de enseñanza-aprendizaje mediante el enfoque blended learning, razón por la cual la cifra obtenida de docentes que lo emplean en su labor diaria no es destacable, por lo que se verifica que el nivel de competencia digital afecta al uso de tal enfoque innovador.

La prospectiva de esta investigación se centra en la necesidad de seguir fomentando la participación del profesorado en programas formativos adaptados a los requerimientos de la educación actual y focalizados en el desarrollo de la competencia digital y en el uso de metodologías emergentes, donde tenga cabida el uso de las TIC, con la finalidad de adecuar la actuación docente a los nuevos paradigmas tecnopedagógicos de una sociedad cada vez más digitalizada.

Como futura línea de investigación se pretende analizar la influencia de variables sociolaborales del profesorado como el sexo, la edad y la experiencia docente sobre el uso del blended learning en el contexto de la Formación Profesional.

\section{REFERENCIAS}

Aarsand, Pål. 2019. "Categorization Activities in Norwegian Preschools: Digital Tools in Identifying, Articulating, and Assessing”. Frontiers in psychology 10: 1-12. https://doi.org/10.3389/fpsyg.2019.00973

Acebedo Afanador, Manuel José. 2017. "La evaluación del aprendizaje en la perspectiva de las competencias". Revista Temas 1, 203-226. https://doi.org/10.15332/rt.v0i11.1756

Agreda Montoro, Miriam, María Angustias Hinojo Lucena y José María Sola Reche. 2016. "Diseño y validación de un instrumento para evaluar la competencia digital de los docentes en la Educación Superior española”. Pixel-Bit: Revista de Medios y Educación 49: 39-56. https://doi.org/10.12795/pixelbit.2016.i49.03

Area Moreira, Manuel, Víctor M. Hernández Rivero y Juan José Sosa Alonso. 2016. "Modelos de integración didáctica de las TIC en el aula". Comunicar: Revista cientifica iberoamericana de comunicación y educación 24 (47): 79-87. https://doi.org/10.3916/C47-2016-08 
Area Moreira, Manuel. 2014. "La alfabetización digital y la formación de la ciudadanía del siglo XXI”. Revista Integra Educativa 7 (3): 21-33.

Arrosagaray, Marcelino, Margarita González-Peiteado, Margarita Pino-Juste y Beatriz Rodríguez-López. 2019. "A comparative study of Spanish adult students' attitudes to ICT in classroom, blended and distance language learning modes". Computers E Education 134: 31-40.

https://doi.org/10.1016/j.compedu.2019.01.016

Avitia Carlos, Patricia e Irma Uriarte Ramírez. 2017. "Evaluación de la habilidad digital de los estudiantes universitarios: estado de ingreso y potencial educativo". Edutec. Revista Electrónica de Tecnología Educativa 61: 1-13.

https://doi.org/10.21556/edutec.2017.61.861

Bliuc, Ana María, Grant Casey, Agnieszka Bachfischer, Peter Goodyear y Robert A. Ellis. 2012. "Blended learning in vocational education: teachers' conceptions of blended learning and their approaches to teaching and design". The Australian Educational Researcher 39 (2): 237-257. https://doi.org/10.1007/s13384-012-0053-0

Cabero Almenara, Julio y Julio Barroso Osuna. 2018. "Los escenarios tecnológicos en Realidad Aumentada (RA): posibilidades educativas”. Aula abierta 47 (3): 327-336. https://doi.org/10.17811/rifie.47.3.2018.327-336

Carmona Herrera, Maribel, Verónica Cruz Rosales y Lydia García Martínez. 2019. "Desarrollo de competencias sociolingüísticas e interculturales en ELE: propuesta didáctica con blended learning”. Revista Comunicación 28 (1): 16-30. https://doi.org/10.18845/rc.v28i1-2019.4442

Castañeda, Linda, Francesc Esteve y Jordi Adell. 2018. “¿Por qué es necesario repensar la competencia docente para el mundo digital?”. Revista de Educación a Distancia 56: 1-20. https://doi.org/10.6018/red/56/6

Cela Ranilla, José María, Vanessa Esteve González, Francesc Esteve Mon, Juán González Martínez y Mercé Gisbert-Cervera. 2017. "El docente en la sociedad digital: una propuesta basada en la pedagogía transformativa y en la tecnología avanzada". Profesorado. Revista de Currículum y Formación de Profesorado 21 (1): 403-422.

De Lange, Peter, Alexander Tobias Neumann, Petru Nicolaescu y Ralf Klamma. 2018. "An Integrated Learning Analytics Approach for Virtual Vocational Training Centers". International Journal of Interactive Multimedia and Artificial Intelligence 5 (2): 32-38. https://doi.org/10.9781/ijimai.2018.02.006

Dummert, Sandra, Ute Leber y Barbara Schwengler. 2019. "Unfilled training positions in Germany-regional and establishment-specific determinants". Jabrbücher für Nationalökonomie und Statistik 239 (4): 661-701. https://doi.org/10.1515/jbnst-2018-0014

Elche Larranaga, Maria y Santiago Yubero. 2019. "The influence of reading habits on the use of internet: a study with university students". Investigación bibliotecológica 33 (79): 51-66. https://doi.org/10.22201/iibi.24488321xe.2019.79.57985 
Fernández Batanero, José María y Alejandro Rodríguez Martín. 2018. “TIC y diversidad funcional: conocimiento del profesorado". European Journal of Investigation in Health, Psychology and Education 7 (3): 157-175. https://doi.org/10.1989/ejihpe.v7i3.203

Fernández Cruz, Francisco José, María José Fernández Díaz y Jesús Miguel Rodríguez Mantilla. 2018. "El proceso de integración y uso pedagógico de las TIC en los centros educativos madrileños”. Educación XX1 21 (2): 395-416. https://doi.org/10.5944/educxx1.17907

Fuentes Cabrera, Arturo, Jesús López Belmonte y Santiago Pozo Sánchez. 2019. "Análisis de la competencia digital docente: Factor clave en el desempeño de pedagogías activas con Realidad Aumentada". REICE: Revista Iberoamericana sobre Calidad, Eficacia y Cambio en Educación 17 (2): 27-42. https://doi.org/10.15366/reice2019.17.2.002

García Martín, Sheila e Isabel Cantón Mayo. 2019. "Teachers 3.0: Patterns of use of five digital tools”. Digital Education Review 35: 202-215. https://doi.org/10.1344/der.2019.35.202-215

Gómez Zermeno, Marcela Georgina, Lorena Yadira Alemán de la Garza y Carlos Manuel Figueroa Orejarena. 2019. "B-learning modality: A strategy to strengthen blended learning vocational training”. Virtualidad Educación y Ciencia 10 (18): $37-51$.

González Bello, Edgar Oswaldo, Guadalupe Aleida Valenzuela Miranda y Adria Velia González Beltrones. 2015. "Significant differences in learning styles with features college student in Mexico". Journal of learning styles 8 (15): 201-221.

INTEF (Instituto Nacional de Tecnologías Educativas y de Formación del Profesorado). 2017. Marco de Competencia Digital. Madrid: Ministerio de Educación, Ciencia y Deportes.

https://aprende.intef.es/sites/default/files/2018-05/2017_1020_Marco-Com\% C3\%BAn-de-Competencia-Digital-Docente.pdf

Larionova, Viola, Ken Brown, Tatiana Bystrova y Evgueny Sinitsyn. 2018. "Russian perspectives of online learning technologies in higher education: An empirical study of a MOOC”. Research in Comparative and International Education 13 (1): 70-91. https://doi.org/10.1177/1745499918763420

López Belmonte, Jesús, Santiago Pozo Sánchez, Arturo Fuentes Cabrera y José María Romero Rodríguez. 2019. "Análisis del Liderazgo Electrónico y la Competencia Digital del Profesorado de Cooperativas Educativas de Andalucía (España)”. Multidisciplinary Journal of Educational Research 9 (2): 194-223. https://doi.org/10.4471/remie.2019.4149

Moreno Guerrero, Antonio José. 2019. "Estudio bibliométrico de la producción científica en Web of Science. Formación Profesional y blended learning”. PixelBit. Revista de medios y educación 56: 149-168. https://doi.org/10.12795/pixelbit.2019.i56.08

Nogueira Leite, Frederico, Eduardo Shigueo Hoji y Humberto Abdala Júnior. 2018. "Collaborative teaching and learning strategies for communication networks". The International journal of engineering education 34 (2): 527-536. 
Pfefferle, Petra Ina, Etienne Van den Stock y Annette Nauerth. 2010. “The LEONARDO-DA-VINCI pilot project "e-learning-assistant"-Situation-based learning in nursing education”. Nurse Education Today 30 (5): 411-419.

https://doi.org/10.1016/j.nedt.2009.09.014

Prendes Espinosa, María e Isabel Gutiérrez Porlán. 2013. "Competencias tecnológicas del profesorado en las universidades españolas”. Revista de Educación 361: 196-222. https://doi.org/10.4438/1988-592X-RE-2011-361-140

Prendes Espinosa, María Paz, Isabel Gutiérrez Porlán y Francisco Martínez Sánchez. 2018. "Competencia digital: una necesidad del profesorado universitario en el siglo XXI”. Revista de Educación a Distancia 56: 1-22. https://doi.org/10.6018/red/56/7

Rodríguez García, Antonio Manuel, José María Romero Rodríguez y Miriam Agreda Montoro. 2019. "Impact of ICT on the teaching of Physical Education: a bibliometric research study”. Education, Sport, Health and Physical Activity 3 (1): 1-14. https://doi.org/10481/53211

Rodríguez García, Antonio Manuel, Juan Manuel Trujillo Torres y José Sánchez Rodríguez. 2019. "Impacto de la productividad científica sobre competencia digital de los futuros docentes: Aproximación bibliométrica en Scopus y Web of Science”. Revista Complutense de Educación 30 (2): 623-646. https://doi.org/10.5209/RCED.58862

Rodríguez Licea, Roberto Alejandro, Blanca Silvia López Frías y Fernando Jorge Mortera Gutiérrez. 2017. "El video como Recurso Educativo Abierto y la enseñanza de Matemáticas”. Revista electrónica de investigación educativa 19 (3): 92-100. https://doi.org/10.24320/redie.2017.19.3.936

Rodríguez-García, Antonio-Manuel, Francisco Raso Sanchez y Julio Ruiz-Palmero. 2019. "Digital competence, higher education and teacher training: a meta-analysis study on the Web of Science". Pixel Bit. Revista de Medios y Educación 54: 65-81. https://doi.org/10.12795/pixelbit.2019.i54.04

Ruhalahti, Sanna, Anne Maria Korhonen y Päivi Rasi. 2017. "Authentic, dialogical knowledge construction: a blended and mobile teacher education programme". Educational Research 59 (4): 373-390. https://doi.org/10.1080/00131881.2017.1369858

Sarmiento Espinel, Jaime Andrés, Adriana Carolina Silva Arias y Edwin van Gameren. 2019. "Evolution of the inequality of educational opportunities from secondary education to university". International Journal of Educational Development 66: 193-202. https://doi.org/10.1016/j.ijedudev.2018.09.006

Tourón, Javier, Deborah Martín, Enrique Navarro Asencio, Silva Pradas y Victoria Íñigo. 2018. "Validación de constructo de un instrumento para medir la competencia digital docente de los profesores (CDD)". Revista Española de Pedagogía 269: 25-54. https://doi.org/10.22550/REP76-1-2018-02 
Tsai, Chia-Wen y Tsang-Hsiung Lee. 2012. "Developing an appropriate design for e-learning with web-mediated teaching methods to enhance low-achieving students' computing skills: Five studies in e-learning implementation”. International Journal of Distance Education Technologies (IJDET) 10 (1): 1-30. https://doi.org/10.4018/IJDET.2012010101

Viñals Blanco, Ana y Jaime Cuenca Amigo. 2016. "El rol del docente en la era digital”. Revista interuniversitaria de formación del profesorado 30 (2): 103-114.

Wheelahan, Leesa. 2015. "Not just skills: What a focus on knowledge means for vocational education". Journal of Curriculum Studies 47 (6): 750-762. https://doi.org/10.1080/00220272.2015.1089942

Para citar este texto:

López-Belmonte, Jesús, Antonio José Moreno-Guerrero, Santiago Pozo-Sánchez y Juan Antonio López-Núñez. 2020. "Efecto de la competencia digital docente en el uso del blended learning en formación profesional". Investigación Bibliotecológica: archivonomía, bibliotecología e información 34 (83): 187-205.

http://dx.doi.org/10.22201/iibi.24488321xe.2020.83.58147 